\title{
EL REINO DE MURCIA EN EL SISTEMA ECONÓMICO MEDITERRÁNEO DE LA BAJA EDAD MEDIA
}

\author{
$M^{2}$ De los llanos Martínez Carrillo \\ Universidad de Murcia
}

\section{SUMARIO}

1. Las rutas y productos mercantiles. - 2. Estratificación de los agentes del mercado.- 3. Los ciclos económicos.- Conclusión.

Cuando el reino de Murcia se incorpora a la Corona de Castilla definitivamente en 1266, la sociedad castellana desarrollaba un proceso avanzado de aristocratización en el que las luchas de bandos fueron una constante en los siglos XIII-XV, analizada por la historiografía regional a partir de Torres Fontes ${ }^{1}$.

En 1984 Menjot exponía su visión de la sociedad murciana entre 1266 y 1492, en la que los rasgos fundamentales eran la implantación de un sistema feudal sobre una sociedad islámica compuesta por campesinos libres y dueños de sus tierras que pagaban un impuesto al estado; en función de esa implantación una minoria de privilegiados fue concentrando en sus manos propiedades urbanas y agrarias, exenciones fiscales, etc. y por debajo de ese patriciado el artesanado fue siempre "notablemente insuficiente e

'Juan TORRES FONTES, Murcia y Don Juan Manuel: tensiones y conflictos. Don Juan Manuel VII centenario, Murcia 1982, pp. 353-383. M de los Llanos, MARTINEZ CARRLLO, Manueles y Fajardos, Murcia, 1985, pp. 270. Juan TORRES FONTES, Fajardo el Bravo, Murcia, 1944, Pp. 179 y Don Pedro Fajardo adelantado mayor del Reino de Murcia, CSIC, 8.a., pp. 322.

"Anuario de Entudion Medievalea", 24 (1994) 
inconsistente" ${ }^{\prime 2}$ Se trataba de una región eminentemente ganadera para aquel tipo de sociedad, en la que los territorios regados en tramos de la cuenca del Segura, suponian una riqueza controlada por el patriciado, pero insuficiente para garantizar el abastecimiento de la ciudad y en muchas ocasiones de otras ciudades y núcleos del reino, sobre todo los cereales, cuya necesidad de importar fue permanente, derivada de las estructuras físicas de la región sobre las que actuaban la política y especulación económicas.

Analizando las posibilidades mercantiles que la ciudad de Murcia ofrecía, Torres Fontes ha incidido en la decadencia gradual de las ferias de Murcia, fundadas en 1266, a lo largo de todo el siglo XIV y el XV, de tal modo que con aspiraciones de internacionalidad en los finales del siglo XIII, quedaron reducidas a unas meras ferias comarcales, no solo las de Murcia, sino las que se celebraban en unas circustancias de aislamiento y peligrosidad aún mayores, como las de Lorca, y otras como las de Jumilla y Orihuela, Elche y Alicante mientras formaron parte del reino de Murcia hasta las sentencias de Torrellas-Elche de 1304-1305, en las que la especiería y los paños de Flandes eran los dos tipos de mercancias que rompían el conjunto de productos estrictamente autárquicas ${ }^{3}$. Es verdad que por la ciudad pasaron muchos mercaderes internacionales de muy diversas procedencias y que al puerto de Cartagena o sus inmediaciones costeras arribaron muchos barcos, sobre todo a lo largo del siglo $\mathrm{XV}$, pero muy pocos fueron los que no se iban después de culminar el negocio concreto que los traía en nombre de alguna casa italiana, valenciana o castellana, en menor medida; los que actuaron durante varias generaciones fueron muy pocos. La ciudad de Murcia nunca dejó de ser un centro de comercio regional sobre el que se intercambiaban materias primas y productos manufacturados, desde la periferia a la ciudad mas importante del reino que era Mur-

\footnotetext{
${ }^{2}$ Denis MlinJoT, Estructuras sociales y modelos de desarrollo en los paises inediterráneos durante la edad inedia: el ejemplo de inercado murciano (1266-1492), "Areas. Desigualdad y Dependencia", Murcia, 1986, pp. 59-64.

${ }^{3}$ Sevilla, 1266-V-19, "Colección de Documentos para la Historia del Reino de Murcia" I, 1963 (en lo sucesivo CODOM), p. 23. Juan TORRES FONTES, Los corredores del comercio murciano en el reincado de Alfonso XI, "MMM" IV (Murcia, 1978), pp. 242-243. José Miguel GuAl, Bases para el estudio de las ferias inurcianas en la Edad Media, "MMM", IX (Murcia, 1982), pp. 9-55, documento 35 .
} 
$\mathrm{cia}^{4}$, y sobre esta base SE producían las exportaciones de materias primas, lana y grana sobre todo, y las importaciones de calidad y alto precio, algodón, azúcar, especias, papel y básicamente cereales que caracterizaron el siglo XV.

\section{LAS RUTAS Y PRODUCTOS MERCANTILES}

La proyección castellana hacia el Mediterráneo se abrió paso con la incorporación del reino a la Corona Castellana a mediados del siglo XIII, mediante la cual se podría utilizar una extensa franja litoral controlada desde los puertos de Cartagena y Alicante, cuyo uso para expediciones mercantiles y corsarias ha desarrollado la bibliografía , en sus consecuencias inmediatas hasta la concreción territorial del reino en los tratados de Torrellas-Elche de 1304-1305'. A partir de entonces el puerto de Cartagena fue la base de salida y entrada de Castilla hacia el Mediterráneo, y los murcianos de Cartagena sus primeros vasallos que, como auténticos mediterráneos, llegaron a las Baleares hacia 1300, islas utilizadas como punto de apoyo para comerciar con el Magreb ${ }^{6}$. Cartagena y Alicante estaban en el centro medular de la mas importante de las tres áreas de pirateria mediterránea que ha marcado Torres Delgado, lo que él llama sector valenciano, que unía Almería con Mallorca y las costas catalanas; los otros serían el sector Málaga-Velez Málaga y el entorno de Gibraltar?.

'M" Llanos MARTINEZ CARRILlo, Revolución urbana y autoridad monárquica en Murcia durante la Baja Edad Media (1395-1420), Murcia 1980, pp. 260-262. Miguel Rondrígue:. LLopIS, Señorio y feudalisino en el Reino de Murcia, Murcia, s.a., pp. 266-274.

SJuan TORRES FONTTS, La delimitación del sudeste peninsular Torrellas-Elche 13041305), Murcia, 1951, pp. 21; los puertos de Cartagena y Alicante en la segunda mitad del siglo XIII, "XI Congresso di Storia della Corona d'Aragona", Palcnno, pp. 347-366. José HINOJOSA MONTAlvo, Artesania y comercio en tierras alicantinas durante el siglo XIII, Alicante, 1985, pp. 143-168. $\mathrm{M}^{2}$ de los Llanos MARTINIZ. CARRIIJO), La sociedad alicantina en la segunda mitad del siglo XIII: la organización de Alfonso X El Sabio, Alicante, 1985, pp. 208-243. Juan Manucl Dill. EsTAL, Conquista y anexión de las tierras de Alicante, Elche, Orihuela y Guardainar al reino de Valencia por Jaime II de Aragón (1296-1308), Alicante, 1982, pp. 423.

${ }^{6}$ Charles E. Dufource, La vie quotidienne dans les ports méditerranéens au moyen âge (Provence-Languedoc-Catalogne), Paris, 1975, p. 96.

'Juan TORRIS FonTlis, Los puertos de Cartagen y Alicante, pp. 347-366. Cristohal TORRFS Di:igaDO, La cosia de Al-Andalus, "V centenario de la incorporación de Salobreña a la Corona Castellana (1489-1989)". Salohreña, 1990, p. 90. 
Para Castilla la pérdida de la baja cuenca del Segura y el puerto de Alicante fue una dura derrota que limitaba sus posibilidades mediterráneas $^{8}$. Alfonso $X$ trató de impulsar y continuar la tradición islámica del puerto alicantino, desde el que se trataron de mantener las rutas derivadas del pasado, que hacen presuponer la existencia de un comercio con Berberia y Baleares, as como otro de cabotaje en el frente costero inmediato, cuyo primer producto fueron los esclavos, de los que el reino de Murcia fue una importante plataforma de extracción desde el siglo XIII, obtenida a través de la actividad fronteriza $y$, de importación cuando las transformaciones agrarias de la segunda mitad del siglo XV precisaron de mano de obra en abundancia que hubo que comprar'.

Análoga política real se desarrolla respecto al puerto de Cartagena desde la concesión del fuero de Córdoba en 1246 y posteriormente en 1257 y 1271; el fomento de actividades corsarias potenciaba la existencia de una armada real y el desarrollo del comercio exterior ${ }^{10}$.

La guerra con Aragon y las posteriores luchas sociales de minorias dinásticas fueron la causa intrapeninsular de que el comercio marítimo castellano apenas tuviese actividad hasta el primer tercio del siglo XIV, cuando Alfonso XI refrendo en 1332 unos acuerdos firmados con Mallorca en 1327. A pesar de la protección real a todos los que armasen barcos en Cartagena, la ciudad fue un misérrimo núcleo marítimo que tenfa 176 vecinos en 1381, de los cuales aún perdió casi la mitad hasta los años 1408-1410. En general la costa murciana estuvo sometida a mediados del siglo XIV a una pirateria, independiente $o$ aliada a los ataques granadinos, que dejaron S!I comarca en situación de sistemático desbastecimiento; de esta situación no se empezo a salir sino a través de una conjunción de factores, tales como la neutralización progresiva de la frontera granadina y la utilización que de su magnifico puerto natural empezaron a hacer como escala las grandes

'Sevilla, 1252-X-25; Monteagudo, 1257-V-10; Murcia, 1271-V-2, "CODOM", III, 1973, pp. 21,56 y 111 .

'Análisis de fuentes originales y publicadas en $M^{2}$ de los Llanos MARTINEZ CARRILO, Rescase de cautivos-comercio de esclavos (Murcia siglos XIV-XV), "Estudios de Historia de España", II (Buenos Aires, 1989), pp. 35-64. M" Teresa FERRER I MALOL, Els italians a terres calalanes (segles XII-XV), "Anuario de Estudios Medievales" (en lo sucesivo "AEM"), 10 (1980), pp. 393466.

${ }^{10} \mathrm{JaCn}, 1246-\mathrm{I}-16$; Monteagudo, 1257-V-6 y Murcia, 1271-V-2, "CODOM" III, Murcia 1973, pp.11, 53 y 111. Juan TORRES FONTES, El concejo de Cartagena en el siglo XIII, "CODOM" IV, Murcia, 1980, pp. XXIX-XXXIV. 
casas de mercaderes en busca de una captura de esclavos o su comercio; todo ello posibilitó que hacia 1474 Cartagena tuviese 280 vecinos y posiblemente unos 600 en $1531^{\prime \prime}$.

Las sucesivas prórrogas de paces abiertas con Granada desde comienzos del siglo XV, propiciaron la llegada a las costas murcianas de muchos barcos; de los ataques granadinos y africanos como el de las 18 galeras llegadas a Cartagena entre 1399 y $1400^{12}$, se pasó a expediciones mercantiles, corsarias o piráticas de distinto signo que afectaban a toda la costa murciana, como las de Pero Niño, Pedro Sánchez de Laredo o Pero de Monsalve estudiadas por Benito Ruano y Ferrer I Mallol. Melis dijo que los últimos años del XIV y comienzos del XV fueron los que propulsaron "...la splendida affermazione della prima grande marina ibérica..." actuante en el Mediterráneo y el Atlántico merced a la apertura dela vía de Gibraltar; en el tramo comprendido entre Alicante y Ayamonte solo los barcos de Málaga y Almería salfan periódicamente hacia Valencia y Mallorca-Ibiza, llegando algunos hasta la propia Génova; los barcos de Sevilla, sin embargo, se relacionaban mas con el Marruecos atlántico y Argelia y en menor medida con Valencia y Baleares ${ }^{13}$.

En ningún caso el puerto de Cartagena era puerto obligado, salvo que las condiciones de algunos negocios concretos a cambio de algún tipo de concesion, lo hicieran factible, como la importación en 1445 de hierro y herrajes destinados a abastecer los núcleos de población levantados contra el nuevo adelantado mayor don Pedro Fajardo ${ }^{14}$; desde los comienzos del XV la intervención genovesa se fue haciendo cada vez mas notoria. Circustancias tan fundamentales como la unión de las dos coronas fronteras y la

\footnotetext{
"Archivo Municipal de Murcia, Actas Capitulares (en lo sucesivo A.c.), 1380-1II-23; Archivo Municipal de Cartagena, A.c. 1528-1537, 1531-VIII-22. Juan T(ORRFS FONTES, Relaciones comerciales enire los reinos de Mallorca y Murcia en el siglo XIV, "Murgetana" (1971), p. 5. M* de los Llanos, Mak'I1Ni:Z. CAkrildo), Población y térinino de Carlagena en la baja edad media, "I Concurso de de Historia de Cartagena 'Federico Casal', Cartagena, 1986, pp. 149-157.

${ }^{12}$ A.c. 1399-XII-2, 1400-11-12 y $111-23$.

${ }^{13} \mathrm{Jose}$ Hinojosa MONTalvo, Piratas y corsarios en Valencia a principios del siglo XV (1400-1409), "Cuadernos de Historia. Anexos de Hispania", 5 (1975), p. 105. M2 de los Llanos MARTfNEZ CARRLLO, Revolución, pp. 226-231. Cartagena es el único puerto murciano citado en los documentos Datini fechados en estos años, Denis MENJOT y Elena CECCHI, Murcie dans le grand commerce intemational á l'orée du XVe siècle, d'apres les archives Datini, "MMM", XV, p. 128. Federigo MEIJs, Wemer Sombart e $i$ problemi della navegazione nel Medio Evo, Milano, 1964, p. 106.
}

${ }^{14}$ A.c. $1445-\mathrm{XII}-13$ y $1446-1 \mathrm{X}-13$. 
posterior conquista del reino de Granada fueron la causa de que en los albores del siglo XVI Cartagena formara parte de una cadena de puertos mediterráneos que conectaba con Almería y Málaga por el $\mathrm{O}$. y Alicante y Valencia por el E., de extraordinaria importancia mercantil en su conjunto, aunque la superioridad mercantil del puerto de Málaga se hace evidente desde entonces ${ }^{15}$.

Es muy significativo que un manual de mercaderes tan tardío como el escrito por Giovanni di Antonio da Uzzano en $1442^{16}$, describa minuciosamente las características de la navegación entre las costas mediterráneas peninsulares y el norte de Africa, demostrando que el puerto de Cartagena, los cabos de Gata y Palos, incluso la isla de Tabarca, eran puntos perfectamente conectados con Argel, Bujfa y otros norteafricanos, amén de Ibiza, resto de Baleares, etc. y, sin embargo, no se dedique un apartado a enumerar productos, calidades y precios como hace con Granada. Solo Cartagena como puerto de salida de la lana y Mazarrón del alumbre y la sosa, aparecen en los finales del siglo XV como puertos con actividad notoria en la documentación valenciana manejada por Guiral y, hacia 1450, Heers no parece contar con él en el sistema de navegación genovés que no era el directo Génova-puerto $\mathrm{X}$ y a la inversa ${ }^{17}$, por el contrario, los grandes barcos iban directamente de Inglaterra a oriente o a la inversa, haciendo escalas "per costeriam"; el negocio estaba en Génova pero los productos no necesariamente todos pasaban por su puerto.

El uso del puerto de Cartagena como puerto de exportación- importación fue en muchas ocasiones puramente circustancial, salvo cuando en el siglo XV se impuso la exportación lanera; Mollenat dice que los mercaderes genoveses establecidos en Toledo canalizaban el mercado de la lana hacia Cartagena, contratando a los carreteros que la transportaban desde un área muy extensa que abarcaba toda La Mancha, marquesado de Villena y reino de Murcia, pagándose el transporte por carretada de mercancia y legua recorrida en valores variables que dependían de las dificultades de

\footnotetext{
is José E. LÓPEZ DE COCA, Cornercio exterior del Reino de Granada, "Actas del II Congreso de Historia Medieval Andaluza, 1982", pp. 357-377.

${ }^{16}$ Giovanni di Antonio UZZANO, Della decima e delle altre gravezze, Lucca, 1766, pp. 187, 240-247.

17Jacqueline GUIRAL-HADZIIOSSIF, Valencia, puerto imediterráneo en el siglo XV (14101525), Valencia, 1989, pp. 37 y 398. Jacques HEERS, Gênes el l'Afrique du nord vers 1450 , "AEM", 21 (Barcelona, 1991), p. 234.
} 
cada momento; Melis, a su vez, subrayó que Cartagena actuó como puerto de salida de lanas, no solo castellanas, sino también aragonesas y del Maestrazgo cuando el puerto de Valencia no resultaba adecuado por razones político-económicas, como ocurrió entre 1397 y $1401^{18}$

En los finales del siglo XV se fueron imponiendo en el puerto de Cartagena las funciones militares, tanto de cara a Italia como al norte de Africa; la salida de barcos con tropas contribuyó a definirlo siendo, tanto un estímulo de consumo para el mercado local, como un pesado lastre para su aprovisionamiento de mercancias, que crecientemente se exigfan a la región, como la preparación de las campañas del Gran Capitán en 1494 obligaron a la saca en grandes cantidades de productos tradicionalmente vedados como el pan y el vino ${ }^{19}$.

Coincidiendo con la concesión del señorío de Cartagena a los Fajardo entre 1464 y 1503 , se encuentran vestigios documentales de un impuesto calificado en Murcia como "mollaje" o "lo del muelle", que era un derecho pagadero por las mercancias que importaban los murcianos, no pagado antes de 1469 y deduciblemente imposición de Pedro Fajardo, al que se resistieron los mercaderes procedentes de Murcia hasta conseguir el acuerdo de permutar el "mollaje" por el derecho de paso por el puerto orográfico de Cartagen $a^{20}$, todo lo cual prueba un cierto grado de actividad.

Como puerto importador se constata en los finales del siglo XV la utilización del puerto alicantino, desde dondo llegaban a Murcia y Villena queso y pescado y se exportaba también materia prima, entre la que Hinojosa señala madera, jabón y otros con destino a Cartagena; era un pequeño comercio regional terrestre o de cabotaje. Con la unidad de las dos coronas Cartagena perdió el casi monopolio de puerto castellano en el mediterráneo; sus mejores condiciones de emplazamiento lo privilegiaban militarmente frente a Alicante mientras que éste segufa una línea comercial ininterrumpida; durante el siglo XVI siguió existiendo comercio de importación de trigo y exportación de lanas por Cartagena, pero fueron los factores

\footnotetext{
${ }^{18}$ Federigo MEUS, La lana de la Spagna mediterránea e della Berberia occidentale nei secoli XIV-XV, "Mercaderes italianos en España (siglos XIV-XVI)", Sevilla, 1976, p. 148. J.P. MOLLENAT, Les coininunications en Nouvelle Castille au XV siecle et au debüt du XVI siècle, "Les communications dans la Péninsule lbérique au Moyen Age", París, 1981, p. 161.

${ }^{19}$ A.c. 1495-1-17, III-16 y III-28.

${ }^{20}$ A.c. 1469-VII-4 y VIII-1; 1490-I-7. M* de los Lanos MARTINEZ CARRILL, La red de caminos del reino de Murcia en los siglos XIII-XV, "II Congreso Intemacional de camineria hispánica" (en prensa).
} 
militares los que prioritariamente dieron movimiento evolutivo a la sociedad local y sus actividades económicas y la proyectaron en esa dirección ${ }^{21}$.

Las dos ciudades extremas del arco terrestre-costero peninsular en el que se insertaba la actividad mercantil ordinaria de Murcia, eran Sevilla y Valencia.

De Sevilla se compraban esclavos, trigo y pescado de calidad traido por mercaderes por mar, pero también muchos otros productos llegados al gran mercado hispalense desde otros lugares y que alli se redistribufan; buen ejemplo de ello y el peligro que el traslado por mar suponía en los finales delsiglo XV, lo proporciona el negocio de compra efectuado en Sevilla y "su tierra" por un grupo de vecinos de Murcia representados por Rodrigo Palazol, Alvaro de Santisteban y Juan Ruiz; adquirieron 56 cahices de trigo toledano, 2 cahices de garbanzos, 7 barriles grandes de atún de badana, 1 pequeño e atún blanco, 42 más de pescada "cecial" o seca, 60.000 sardinas blancas mas ropa y armas que embarcaron en una carabela del Puerto de Santa María para trasladar a algún puerto murciano; pero a la altura del cabo de Gata les salieron dos naos pilotadas por dos sevillanos, "La Garza" al mando de Diego del Puerto y "Falconete" a cargo de Melchor, que se apoderaron de todo el cargamento, valorado en $170.000 \mathrm{mrs}$. y huyeron con él refugiándose en Almería ${ }^{22}$.

$\mathrm{La}$ intensidad del comercio con Sevilla fue constante a lo largo de toda la baja edad media, tanto en los negocios que alli hacían gentes procedentes de Murcia como los que de pasada hacia otras plazas, no tanto en Murcia, hacian los comerciantes sevillanos donde, como los de cualquier otra procedencia, obtenf́an seguro concejil ${ }^{23}$.

\footnotetext{
${ }^{21}$ José HINOJOSA, El puerlo de Alicante durante la baja edad imedia, "Anales de la Universidad de Alicante. H" Medieval", 4-5, pp. 155-157. Francisco CHACÓN Jimí́ntZ, Murcia en la centuria del quinientos, Murcia, 1979, pp. 56, 154, 171, 295. Angel Luis MoluNA MolJNA, Proyección inediterránea del Reino de Murcia en la Edad Media, "MMM", XVII (1992), pp. 59-75.

${ }^{22}$ A.c. 1473-1-2; este acto pirático tuvo largas consecuencias judiciales y represalias en Murcia a vecinos de Sevilla, A.c. 1477-VIII-2 y 1480-111-14. Juan TORRES FONTES, Estampas medievales, Murcia 1988, pp. 16-18. A. MAC KAY, Comercio y mercado interior y la expansión económica del siglo XV, "Actas del II Congreso..", Sevilla, 1982, p. 109. Miguel Angel LADERO QUESADA, Las almadrabas de Andalucia (siglos XIII-XVI), "Boletín de la Real Academia de la Historia, CXC, cuademo III (1993). $M^{2}$ de los Llanos MARTINEZ CARRLLO, Revolución, p. 342.

${ }^{23}$ A.c. $1439-111-31$.
} 
Ocasionalmente, las fuentes también denuncian la venta de caballos en Sevilla a la oligarquía murcina; en 1468 a cambio de trigo local, cosa rara; con ocasión de los tumultos ocasionados en la ciudad a la muerte del infante don Alfonso y las dispetas por la sucesión al trono, el negocio se hizo por mar en una fusta en la que se transportaron a Sevilla 40 cahices de trigo como forma de pago, porque no habla otra moneda disponible ni aceptable para comprar un mínimo de seis caballos ${ }^{24}$.

La actividad murciana en Valencia era mas bancaria que estrictamente comercial, mas tluida de lo que pudiera ser como impedimento la existencia de una frontera separadora; incluso el movimiento de personas y el ejemplo de influencias técnicas, artísticas y rituales se deja sentir continuamente ${ }^{2 s}$; buena brueba de que ese flujo de gentes no era desdeñable fue la prohibición de conexión alguna de entrada o salida desde o hacia Valencia, cuando en aquella ciudad y su tierra se desataba la peste $e^{26}$.

No era tanto un mercado en el que Murcia se abasteciera de productos deficitarios, como una plaza en la que se buscaba el préstamo o la acción bancaria oportuna dirigida hacia Granada que utilizaba la ciudad de Murcia a través de Orihuela, como punto de apoyo o los puertos murcianos como vía de paso hacia Almería; el comercio directo no era tanto ValenciaMurcia como Valencia-Almeria ${ }^{27}$. Magnifica prueba de los innumerables préstamos buscados desde Murcia en la banca valenciana fue el conseguido a través de genoveses para poder comprar en Barcelona un conjunto de armas de altísima calidad por valor de 1.000 florines, para equipar a los Manuel entre 1392 y 1393, y mas modestamente los 8.000 mrs. que el mercader valenciano Martín Ruiz "dio en cambio" a cpbrar en Roma, al

\footnotetext{
${ }^{24}$ A.c. 1468-IX-17 y X-15. Juan TORRES Fontes, Don Pedro Fajardo, pp. 109-115.

${ }^{25}$ Lo mismo el orden que los gremios debían seguir en las procesiones, ante la duda murcina, se marca por el racional de Valencia, A.c. 1431-IV-17, Maria MARTINEZ MARTINEZ, La industria del vestido en Murcia (ss. XIII-XV), Murcia, 1988, pp. 45 y 346, que se intemaba, en "la casa, de los Inocentes de Valencia" a la mujer del armero Guillén, "que es loca", A.c. 1476-IX-28.

${ }^{26}$ A.c. $1459-$ VII-10, X-20 y $1478-\mathrm{VI}-6$.

${ }^{27}$ En 1428 Juan Soriano y otros mercaderes valencianos y posteriomente Antonio Marín, de Orihuela, recibieron carta de seguro del concejo murciano para traer mercancias hasta los Alcázares y desde alli "sacar retomo", A.c. 1428-III-14, VI-29 y 1472-X-30. J. Hinojosa da primacía a la plaza de Almeria sobre Málaga en el comercio valenciano practicado por valencianos, cristianos y musulmanes, por la menor presencia de genoveses en Almería que en Málaga, Las relaciones entre Valencia y Granada durante el siglo XV: balance de una investigación, "Estudios sobre Málaga y el Reino de Granada en el V Centenario de la conquista", J.E. LÓPEZ DE COCA, editor, Málaga, 1987, pp. 99 y 102.
} 
concejo de Murcia para ganar una bula papal que aprobase la transformación franciscana a la observancia ${ }^{28}$. En distinta gradación, el nexo Valencia-Génova era a través de los judios en escala menor, la cobertura bancaria de la sociedad murciana, por ello no solo se usaba su moneda sino que se guardaba y vigilaba; en 1492 se ordenaba aceptar el real de Aragón igual que el de Castilla, excepto los que fuesen claramente de plomo y en 1501 , los rumores llegados de que "çiertos dinericos" sin valor en Valencia eran introducidos en Murcia exprofesamente, obligó a la intervención concejil en su contra ${ }^{29}$.

Por tierra, el camino real castellano $0^{30}$ era la vía por la que pasaban en uno $u$ otro sentido las mercancias en dirección a La Mancha con centro en Toledo, desde donde se aprovisionaba la ciudad de metalurgia para uso diario de mas calidad que la propia, y el Marquesado de Villena, comercio que se vio potenciado por la existencia de ferias en todo el territorio, estimuladas por la propia nobleza a partir del triunfo de Enrique II, valgan como ejemplo las de Almagro fundadas en $1374^{31}$ y las de Albacete, muy activas ya por ese mismo año, así como la reactivación de otras anteriores correspondientes a lo que Ladero llama el primer ciclo ferial, entre las que estaba la de Albacete aunque de ellas no haya noticias directas hasta 1374.

En 1984 publiqué unas noticias concretas e inéditas entonces, de 1374 y 1376, acerca de la actividad de las ferias de Albacete, en relación tanto con Murcia como con el temor reivindicativo de Chinchilla a perderlas; hablé entonces de las consecuencias que para ambos núcleos tuvo la decisión del marqués de Villena de transformar en villa a Albacete en 1375, disputas por el control economico de las ferias que podían derivar en emigración de Chinchilla a Albacete con el consiguiente crecimiento demo-

\footnotetext{
${ }^{28} \mathrm{M}^{\circ}$ de los Llanos MARTtNEZ CARrJLL, Manueles, pp. 168-194; A.c. 1464-VII-17. La importancia de las armas catalanas está estudiada por Martí de RIQUER, L'amès del cavaller. Armes $i$ armadures calalanes medievals, Barcelona, 1969. En los comienzos del siglo XVI se seguía importando hierro, acero y productos acabados, A.c. 1502-V-7, elaborados en Valencia con hierro de Vizcaya, Miguel Gual Camarena, El hierro en el medievo hispano, "La mineria hispana e iberoamericana", León, 1970, pp. 286-287.

${ }^{29}$ A.c. 1492-III-8 y 1501-III-2.

${ }^{30} \mathrm{M}^{*}$ de los Llanos MARTINEZ CARRILLO, La red de caminos

${ }^{31}$ Miguel A. LADERO QUESADA, Las ferias de Castilla. Siglos XII-XV, 1994, pp. 78-83 y L.R. VILLEGAS DiAZ, La fundación de las ferias de Almagro, "Homenaje a la Memoria del Profesor Dr. Emilio Sáez", I, Barcelona, 1988, p. 282.
} 
gráfico de la nueva villa por su mejor situación; planteé la hipótesis de que sus orígenes se remontarían a la fundación de la villa en época de don Juan Manuel, etc. ${ }^{32}$, de tal modo que se puede considerar la primera investigación hecha sobre este tema, sin que se haya aportado nada nuevo sobre su cronología y lucha por la supervivencia.

A partir de aquellos hechos la presencia de vecinos de Albacete vendiendo en Murcia "sus viandas" excedentarias a comienzos de verano era ya una costumbre en el siglo $\mathrm{XV}^{33}$, así como las murcianas en aquellas ferias, comprando sobre todo ganados para abastecimiento de carne de la ciudad. Hellín punto clave de la ruta hacia pagar una serie de cargas fiscales que los mercaderes eludían pasando directamente desde Tobarra a Minateda, las etapas anterior y posterior, de modo que la reclamación de su concejo ante Enrique III ${ }^{34}$ trat $\delta$ de cortar el fraude originado con la rectificación del itinerario normal.

Además de Hellín, los puertos de La Losilla y Mala Mujer representaban los pasos fiscales fundamentales en el reino ${ }^{35}$; sin embargo, salvo en el trigo y la lana, los intercambios terrestres siempre significaron menos que los procedentes del Mediterráneo, aunque los pequeños mercaderes del interior de Castilla nunca faltaron de tránsito por Murcia, como Juan Daza y Andrea, vecinos de Valladolid, que hicieron valer en Murcia exenciones reales que en ella se les pretendían cobrar ${ }^{36}$.

El camino castellano con meta en el puerto de Cartagena fue a finales del siglo XV una de las dos rutas de exportación de la lana, la otra desde el Sistema Ibérico llegaba a Valencia, quedando a elección del comprador la mas adecuada, generalmente la murciana, que al no atravesar mas que territorio castellano evitaba el pago de derechos aduaneros en ambos

\footnotetext{
${ }^{32}$ A.c. 1374-VII-29 y IX-15 y Carta de Chinchilla, 1376-IV-22, inserta en A.c. 1376-IV26 que publiqué como apéndice documental, $M^{*}$ de los Llanos MAR'ÍNi:Z CARRILIO, La población albaceteria en la segunda mitad del siglo XIV. "Congreso de Historia de Albacete" II, 1984, pp. 109-120. Los documentos han sido posteriomente publicados con distintos criterios paleográficos en "CODOM", XIV, 1985, pp. 36, 48 y 160.

${ }^{33}$ A.c. 1463-VII-16.

${ }^{34}$ Burgos, 1405-VIII-IV, inserto en A.c. 1405-IX-12.

35Juan TORRES FONTES, Puerto de La Losilla, portazgo, torre y arancel, "MMM", IX (1982), pp. 57-85 y Francisco VEAS ARTESI:ROS Montazgo y portazgo en el Marquesado de Villena: el acuerdo de Albacete de 1384, "Congreso de H" de Albacete", pp. 93-108.

${ }^{36}$ A.c. 1487-V-24.
} 
reinos $^{37}$. Asf como también ruta de penetración del pescado de la costa regional, que cuando se podía garantizar por encima de los ataques piráticos procedentes de puertos muy próximos como Vera, o muy lejanos como Barcelona e $\mathrm{Ibiza}^{38}$, se vendía en el mercado del reino murciano y aún en el interior de Castilla mas allá de Hellín.

\section{ESTRATIFICACIÓN DE LOS AGENTES DEL MERCADO}

Torres Fontes y Molina Molina, en dos trabajos complementarios, estudiaron el entramado que unía a los genoveses con el puerto de Cartagena y el concejo de Murcia, así como su actividad mejor documentada, el préstamo en relación con los judios y su activisima participación en una serie de negocios de entre los que destacan la importación de tintes y textiles de gran calidad que ha estudiado Martínez Martínez ${ }^{39}$.

Es posible complementar las actividades de las principales familias genovesas, muy pocas, desde su implantación en la segunda mitad del siglo XIV y en relación con los dos centros económicos mas importantes de su actividad que eran Sevilla y Valencia, conectados a los cuales actuaban. En el siglo XIV Cataneo y Usodemar fueron las dos únicas familias implantadas en la ciudad de forma permanente, algunas veces asociados entre sf.

Los Cataneo tuvieron una actividad ininterrumpida por lo menos durante un siglo. Las primeras documentaciones surgen en plena conflagración social de Manueles y Fajardos, cuando Jacomo Cataneo en 1394 actuó como banquero de los Manuel asociado a los judios; por su mediación se compró trigo y orfebrería de plata en Valencia para pagar al rey parte de las monedas derramadas entre 1392 y 1393; las circustancias adversas para su actividad le hicieron utilizar junto a Polo Usodemar un per-

\footnotetext{
${ }^{37}$ Jacqueline GuIRAL-HADZIIOSSIF, Valencia, p. 112.

${ }^{38}$ A.c. 142I-XI-15; 1426-I-2, I-8; 1430-VIII-26; 1427-V-17; 1462-III-2; 1439-VIII-1; 1458-IX-2 y 1459-I-2. Juan TORRES FONTES, La pesca en el litoral murciano durante la edad media, "Nuestra Historia", Cartagena, 1987, pp. 113-127.

${ }^{39}$ Juan TORREs FonTES, Genoveses en Murcia (siglo XV) y Angel Luis Mouna MouNA, Mercaderes genoveses en Murcia durante la ápoca de los Reyes Católicos (1475-1516), "MMM", II, pp. 69-168 y 277-312 respectivamente. Posteriormente María MARTINEZ MART1NEZ ha analizado las bases estructurales y los cambios coyunturales del comercio textil, La industria del vestido, pp. $88-148$ y La seda en Murcia: decadencia y reactivación de una actividad musulınana. "La ciudad islámica", Zaragoza, 1991, pp. 465-471
} 
miso real para abandonar el reino, análogo al que les habra sido entregado a los genoveses de Sevilla, lo cual convierte en cuestión a investigar las consecuencias que para los genoveses pudieran haber tenido las agresiones sufridas por los judios, dado que el permiso de Juan I tenía fecha de 1389 y no habla sido necesario usarlo hasta $1394^{40}$. SI se decidieron a marcharse a Génova renunciando a unos negocios tanto bancarios como comerciales, tal como se acredita en Sevilla con el mercado del trigo, es que poco tenian que hacer en Murcia. En 1412, de los 12 genoveses existentes en Sevilla tres eran Cataneo o Castaño, Pelegrín y su hermano Jácomo y Nicoloso $^{41}$. No hay otras noticias de entidad mercantil sobre ellos salvo las referentes a los que permanecieron integrados en la sociedad local, como Francisco y Bertolín en los años veinte y cuarenta, hasta que los hermanos Gabriel y Simon, habitantes de la ciudad en 1460 continuaban con el comercio de importación de trigo y estaban ya plenamente inmersos en el de los tintes, en estrecha relación tanto con la casa sevillana de Anselmo Cataneo como con Valencia donde esta familia es una de las que practicaban el préstamo y el cambio monetario ${ }^{42}$.

Los Usodemar son un claro ejemplo de familia de mercaderes que a través de los negocios llegaron a entroncar con algún sector de la nobleza castellana, creo que el centro primario de su actividad fue el mercado de esclavos y la banca ligada a los Bocanegra; entre 1405 y 1406 Percival Usodemar prestó a la ciudad cantidades sucesivas que llegaron a 50.000 mrs. en 1406, cuando ya se le debian mas de 16.000, con dos objetivos, hacer frente a la construcción del Alcázar Nuevo de la ciudad y devoluciones a los Bocanegra, tesoreros del Rey, con importantes negocios en Ori-

\footnotetext{
${ }^{40}$ A.c. 1394-VII-28; Toro, 1389-IX-26, inserta en 1394-X-1. M' de los Llanos MARTINEZ CARRILL, Manueles, pp. 163-189 y Los judios de Murcia a través de fuentes municipales. Hipótesis de trabajo, "Espacio, Tieınpo y Fonna" (en lo sucesivo "ETF") (Madrid, 1993), pp. 159-174.

"Antonio, COLlANTES DE TERAN, Sevilla en la baja edad media. La ciudad y sus hombres, Sevilla, 1977, pp. 215-216. Jacques HEERS, Los genoveses en la sociedad andaluza del s. XV: origenes, grupos y solidaridades, "Actas del II Coloquio de Historia Medieval Andaluza", Sevilla, 1982. Manuel GONZÁLZ JIménEZ, Genoveses en Sevilla (Siglos XIII-XV), "Actas del II Coloquio Hispano-Italiano sobre la presencia italiana en Andalucia (ss. XIVXV), Sevilla, 1985, pp. 115.130. Miguel Angel LADERO QUESADA, Los genoveses en Sevilla y su región (siglos XIII-XVI). Eleinenios de permanencia y arraigo, "Los mudejares de Castilla y otros estudios de Historia Medieval Andaluza", Granada, 1989, pp. 283-312.

${ }^{2}$ A.c. 1460-XI-18) y XII-11. Juan TORRES FONTES, Genoveses, pp. $81-87$ y 111. D. IGUAL LUIS, Valencia y Sevilla en el sisterna econónico genovés de, finales del siglo XV, "Revista d'Historia Mcdieval" (Valencia, 1992), pp. 91-102
} 
huela. Verlinden recoge la llegada a Génova en 1396 del navío de Nicoloso Usodimare con esclavos procedentes de Crimea, posteriormente redistribuidos por Europa, y en la Murcia de finales de ese siglo Torres Fontes ha identificado hasta seis Usodimar en Murcia ${ }^{43}$; uno de ellos, Millán Usodemar se identificó plenamente con el patriciado local, llegando a ser regidor y posteriormente alcalde de la ciudad en 1410, demostrando un filojudaismo muy notable cuando llegó la gran prueba de las predicaciones de san Vicente Ferrer en 1411, lo cual no era mas que un reflejo de que judios y genoveses eran dos sucesivos eslabones en la actividad mercantil, habiendo intercambios entre ellos que en 1422 se detectan muy favorables a un notable coverso de 1411, Lope Alonso de Lorca, que prestó 100 florines a Perçival Usodemar para los negocios que éste tenfa en Valencia-Génova, tal como testificaba la documentación que su hijo, Gregorio Usodemar, había recuperado al ser devuelta la deuda por su padre despues de retornar del viaje que habra hecho a Génova ${ }^{44}$. A nivel local alguno se identifico con la élite, Millán, acabó con la práctica de los grandes negocios que caracterizaron a los Usodimare en sus orígenes internacionales e hispánicos. Dos de ellos, Polo y su hijo Próspero están identificados en Murcia entre 1376 y 1396 y en Sevilla en 1412 y en Murcia en 1424-1425, lo que parece indicar unas estancias temporales de la familia a tenor de las indicaciones que marcaba la casa matriz.

En la segunda mitad del siglo XV los genoveses dominantes en los negocios murcianos de cara al exterior fueron los Spínola, Negro y Rey.

Los Spínola actuaron como importadores de paños y tintes a partir del establecimiento de Simón y Bartolomé Spínola en los años cincuenta, cuando en Málaga había unos doce Spínolas, los mas numerosos de toda la colonia de aquella ciudad según Ladero ${ }^{45}$. En Murcia obtuvieron permisos de establecimiento que exclufan una posible competencia con los Cataneo y

\footnotetext{
${ }^{43}$ Charles, Verunden, L'esclavage dans la Péninsula Iberique an XIVeme sizcle, y Aspects quantitatifs de l'esclavage méditerranéen au bas Moyen Age, "AEM", 7 (1970-1971), p. 586 y 10 (1980), p. 770. Juan TORRES FONTES, Genoveses, pp. 81 y 87. $M^{\top}$ de los Llanos MARTINeZ CARRILL, Rescate de cautivos-comercio de esclavos (Murcia siglos XIV-XV), "Estudios de Historia de España", II, Buenos Aires, 1989, pp. 35-64.

4A. C. 1427-XI-8; M" de los Llanos MARTINEZ CARRLLO, Revolución, Pp. 50, 55 a 57, 74, 107, 178, 267, 281. Percival Usodemar puede ser el mismo que GuIRAL denomina Pere Anvall de Uso di Mari, Valencia, p. 100 y Collantes, Sevilla, p. 215. 43.

${ }^{45}$ Miguel Angel LADERO QUeSADA, Granada. Historia de un pais islámico (1232-1571), p.
} 
Casanova, que en 1460 tenían monopolizado el abastecimiento de tintes. Mientras las licencias de instalación de estos Spínola y otros genoveses con ellos, fueron concedidas por el concejo, en 1470 era el adelantado mayor Pedro Fajardo quien otorgaba uno mucho mas profundo a Juan Bautista Spínola y Antonio Calvo, para que desde Cartagena pudieran comerciar con toda Castilla, introduciendo tanto tintes como paños de calidad, al tiempo que les aseguraban libertad para exportar todas las lanas y cueros que a Cartagena trajeran, de Castilla o de otros reinos, con referencia indudable a Granada; alli controlaban la "Sociedad de los frutos del Reino de Granada", basada en la exportación de higos, pasas y mas tarde seda y azúcar, cordobanes, y la importación de especias y paños florentinos, en clara dependencia de sus centros de Génova y Sevilla, entre los cuales Cartagena, Alicante y Málaga eran el centro de un área de aprovisionamiento que se complementaba con el norte de Africa, a la vez que puerta de entrada de los productos italianos y orientáles ${ }^{46}$.

La familia de los Negro es una de las cinco familias genovesas actuantes en Barcelona en la década 1484-1494 y en general en toda la Corona de Aragón, en relación recíproca con Génova, Valencia y el resto del Mediterráneo occidental; su actuación en Murcia ha sido relacionada por Molina con los conciertos concejiles, hasta llegar en algunos casos a su avecindamiento, en un proceso similar al que vivieron sus coetáneos los Rey ${ }^{47}$. Mucho antes de todo ello, Simón Negro y otros tres genovesesmoradores, miembros de distintas compañfas vendfan el pastel en precios concertados entre ellos para obligar a los tintoreros a no poder trabajar con los precios concejiles, además de proporcionar un pastel de peor calidad del que vendían en otros puertos. Galeoto de Negro segura vendiendo pastel y obtenía en 1467 licencia para sacar por Cartagena una "baleta" o fardo de paños de Cuenca rumbo a Génova, en un extraordinario ejemplo de tráfico precolonial; en 1472 él mismo, con Tadeo de Negro, Simón Cataneo y

${ }^{46}$ A.c. 1497-IX-23. G. di A. UZZ.ANO, Della cima e delle altre gravezze, p. 187. Juan TORRES FONTES, Genoveses, docuinentos XIV, XV, XVII. J.E. LOSPIEL IDE COCA, Málaga "colonia" genovesa (siglos XIV-XV), "CEM", I (1973), pp. 135-144. R.G. PIIINAIDO SANTALLLA y J.E. LOPEZ DE COCA, Historia de Granada II, Granada, 1988, p. 303 y D. IGUAL LUIS, Válencia y Sevilla, pp. 96-98. María MARTINIEZ MARTINIE, Producción de azúcar en Murcia: un proyecto fracasado del siglo XV. "En 1492: lo dulce a la conquista de Europa", Granada, 1994, pp. 147-158.

${ }^{47}$ Angel Luis, Mouna Mouna, Mercaderes genoveses, pp. 283-284 Documento I y D. IGUAL LUIS, Valencia y Sevilla, p. 89. 
Juan Imperial se comprometían a traer trigo de Andalucia ${ }^{48}$. Su actividad de financieros concejiles solo era un complemento dentro del gran arco mercantil en el que se movían desde Andalucía a Génova, en el cual el reino de Murcia era un punto de apoyo para sus actividades. Cartagena y el reino de Murcia les interesaban como conexión en su gran hinterland y, sobre todo, en los finales del siglo XV por la extracción del alumbre, como demuestra la llegada de un nuevo miembro de la familia, Juan Antonio de Negro, al que el concejo investigaba en 1496 porque utilizaba la fuerza de su casa para cobrarse a través de exportaciones ilegales y ocupación de alguna heredad, deudas no pagadas; su relación con Nicolau y Francesc del Nero, instalados en Valencia, que enviaban el alumbre a Flandes en naves vascas, es evidente ${ }^{49}$.

De todos los genoveses en Murcia, los Rey fueron la familia implantada con mayor continuidad desde los años treinta del siglo $\mathrm{XV}{ }^{\text {so }}$, en primerísima relación con el abastecimiento de las tintorerías desde 1431, con la condición de que quién no quisiere comprar los tintes a Fernando Rey, asociado a micer Anfrión, no tenfa mas remedio que comprarlos en Valencia, lo cual dejaba el mercado local de tintes en manos de Francisco Rey durante los seis años que duró el contrato; no llegó a estar permanentemente instalado en la ciudad, pues en 1434 regresaba a Génova y dejaba como sus factores a Polo Cataneo y Bernabé de Ricobón que era su sobrino; los negocios en el reino le interesaron al punto de volver en 1437 para asociarse con otros genoveses que ya eran habitantes de la ciudad, Simon Negro, Felipe Sagala y Gabriel Lerta, a través de los cuales hacía llegar mercancías no especificadas a Toledo y Villarreal. El proceso de asentamiento que ya se aprecia en él, abre un paréntesis hasta los años setenta en que Baltasar Rey empezaba a configurarse como el gran exportador de trigo y de alumbre del reino con destino a Italia por Cartagena, con ganancias personales suficientes para invertir en tierras y ocupar un lugar muy prominente en el fin de siglo regional. El comercio del alumbre murciano por los Rey equiparaba en importancia en 1488 al italiano de Piombino, que en

\footnotetext{
${ }^{48}$ A.c. 1438-V-2; 1466-VI-28; 1467-V-2; 1472-V-23.

${ }^{49} A . c .1496-V I-18$. Jacqueline GuIRAL-HADZIIOSSIF, Valencia, p. 402.

soJuan TORRES FONTES, Genoveses, pp. 107-109
} 
años sucesivos llegaría hasta Flandes relacionado con la saca clandestina de oro que se dio en el banco de Valencia a comienzos del siglo $\mathrm{XVI}^{51}$.

Muchos otros genoveses pasaron por tierras murcianas, aunque en relación mas subordinada a sus casas de orígen y mas aislados que los anteriormente expuestos, así Nicolás Escarzáfigo, agente en tierra murciana de los negocios de su familia en Cataluña analizados entre 1394 y 1408 por Ferrer i Mallol, quien ha establecido que traficaban con Africa, Granada y las Canarias, los cual los vincula al tráfico de esclavos. También en la segunda mitad del siglo XIV micer Guido de la Vetula; Rafael, Carlos y Jerónimo Vivaldo en 1443, Nicolás Gambo que además de tintas importaba también papel en 1460-1462 y otros muchos; pero sus negocios murcianos y sus habituales faltas de escrúpulos en contradicción con las de la propia burguesía regional hacian que muchos fracasaran y otros presionaran para conseguir mejores condiciones, amenazando con dejar la ciudad, como Domenego, que entre 1471 y 1472 apenas pudo aguantar acosado por las deudas que no consegura cobrar habiendo vendido a crédito ${ }^{52}$.

Otros italianos actuantes en Murcia no dejaron huellas en la documentación regional, como Benvenuto Michi, enviado por los Datini a esta ciudad; la parquedad de los documentos del archivo de esa casa que hacen referencia a Murcia es muy significativa del papel que la ciudad y el reino jugaban en el Mediterráneo, ninguna carta de cambio, ningún contrato de seguro de barco que vincule la costa Murcia y un puerto italiano, solamente ocho cartas escritas en Murcia por un corresponsal de la firma, de las que solo cuatro tienen interés para el comercio de la ciudad en relación con la casa Datini que sirve como muestreo en este caso, cinco van dirigidas a Valencia, dos a Barcelona y una a Mallorca donde la firma tenía filiales ${ }^{53}$. La "Pratica di mercatura" para uso de la casa escrito entre 1380 y 1396 según Ciano y entre 1385 y 1386 según Gual, la época mas dura de las

"A.c. 1494-IV-29. Alfonso Franco SILVA, El aluimbre inurciano, "MMM", VI (1980), p. 246. Jacqueline GUIRAL-HADZIIOSSIF, Valencia, pp. 400-401 y Miguel Angel LADERO QUESADA, El banco de Valencia, los genoveses y la saca de oro casiellana. 1500-1503, "Homenaje a la Memoria del Profesor Einilio Sáez, I, Barcelona, 1988, p. 583.

${ }^{32}$ A.c. 1443-VII-9; 1460-XII-9; 1462-II-27; 1471-IX-24 y 1472-VIII-8. M' Teresa FERRER I MALLOL, Mercanti italiani nelle terre calalane: gli alessandrini (1394-1408), "Estrato della Revista di Storia Arte e Archeologia per le province de Alessandria e Asti", 1966, pp. 18-19.

${ }^{33}$ Denis MENJOT, Estructuras sociales, p. 63 y Murcie dans le grand commerce, pp. 123137. pp. 123-137. 
tensiones de suplantación entre los viejos Manuel y los nuevos Fajardo ${ }^{54}$, no cita ni una sola localidad de la costa murciana, ni los precios de ningún artículo de los que tradicionalmente se han considerado como exportados por ella a traves de Cartagena; la pobreza del reino unida a una profunda revulsión social explican el desinterés de los Datini y tantos otros, por el reino en el siglo XIV. En los mismos años se recopiló el manual catalán estudiado por Gual, 1385, y en todo él no se cita Murcia mas que como centro exportador de higos y grana, con referencia a los primeros como corrientes $^{5 s}$. Ni en uno ni en otro manual se cita, ni siquiera Cartagena, prueba de la inoperancia del puerto para el comercio marítimo hasta el siglo XV, salvo en el caso de los esclavos y sí acaso el trigo, y la implantación en el gobierno regional de los Fajardo como adelantados mayores con la subsiguiente formación de una estructura social puramente feudalizada en tiempos de premercantilismo avanzado.

\section{LOS CICLOS ECONÓMICOS}

La política monetaria castellana desde la entronización de Enrique II estuvo condicionada por constantes necesidades de devaluación seguidas de carestías que incidieron gravemente sobre todo el mercado; desde la primera reorganización de la moneda de 1369 no se hizo mas que ir superponiendo una acuñación devaluatoria tras otra, obligadas por las circustancias políticas, interiores y exteriores ${ }^{\mathrm{st}}$.

Las consecuencias en el comercio local e internacional hay que analizarlas en su incision sobre dos coordenadas inamovibles, la realidad fronteriza del reino y la tradicional facilidad de la moneda aragonesa para imponerse en territorio murciano, por tener mas valor intrínseco y porque el comercio con esta región castellana le resutaba favorable. A partir de

\footnotetext{
${ }^{4}$ Cesare CIANo, La "Pratica di inercalura" Datiniana (secolo XIV), Milano, 1964, p. 12. Miguel GUAL CAMARENA, El primer imanual hispánico de imercaderia (siglo XIV). Barcelona, 1981, p. 8. M' de los Llanos MARTINEZ CARRILL, Manueles.

${ }^{35}$ Miguel Gual Camarina, El primer inanual, pp. 236 y 239.

${ }^{56}$ Julio VALDEÓN, Las reformas monetarias de Enrique II de Castilla. "Homenaje al profesor Alarcos García", Valladolid, 1965-1966 y Reflexiones sobre la crisis medieval en Castilla, "Estudios dedicados al profesor Angel Ferrari", Madrid, 1984. Miguel Angel LADERO QUESADA, Fiscalidad regia y génesis del Estado en la Corona de Castilla (1252-1504), "ETF", 4 (Madrid 1991), p. 99.
} 
entonces apenas circulaba por Murcia moneda castellana y los precios subieron en los productos fundamentales, pan y vino, acentuando las carencias intrínsecas, al tiempo que Pedro IV inundaba el reino de falsos reales y cruzados, como parte de su política de intento de recuperación del mismo. Las prohibiciones de exportación aduanera se endurecieron hasta llegar a una casi absoluta autarquía que no era proclive a la implantación de mercaderes extrajeros que no favorecieran a la nueva oligarquia; el problema era especialmente grave en los cereales aunándose los problemas político monetarios con la larga serie de malas cosechas, "por quanto los que tienen el pan puesto e non lo quieren sacar a vender e por esta razon la conpaña del pueblo que lo an a conprar non lo fallan a presçio alguno"s7.

La progresiva situación de enfrentamiento entre Manueles y Fajardos como secuela regional de la guerra civil, el empobrecimiento del reino y la desconfianza en la propia moneda favorecieron el comercio clandestino en general y en mayor medida con Aragón; en 1380 la única moneda utilizada en Murcia con fluidez eran los "realejos de Aragón y el florín aragonés como moneda fuerte acaparó el mercado regional con un valor ascendente que pasó de $21 \mathrm{mrs}$. en esa época a los 35 de $1420^{58}$ a tenor del mayor endeudamiento social y concejil y las consecuentes mayores exigencias de los banqueros; oro y plata castellanos no solo escaseaban sino que eran objeto de un contrabando casi incontrolable, todo lo cual bloqueó los intentos mercantiles de instalación continuada de gentes procedentes de Valencia, genoveses o no, siendo los pequeños intercambios excedentarios el comercio mas evidente. La prueba de que se trataba de un contrabando interregional, procedente en su mayoría de excedentes domésticos la proporciona el resultado de las pesquisas aduaneras de Enrique II de 1373 y 1375, de las que se deduce que los contraventores de las leyes de las sacas

\footnotetext{
${ }^{57}$ A.c. 1371-X-8 y XII-6. Agustín, El proceso de consolidación del Reino de Murcia en la Corona de Castilla (1243-1375, "Studia Historica", pp. 16-17. Angel Luis Moluna MouJNA, Repercusiones de la guerra casiellano-aragonesa en la economia murciana (1364-1365), "MMM", III (1977), p. 130, nota 50. M de los Llanos MARTINEZ CARRIIJO, Manueles, pp. 85-106 y Una economia tentacular. La relación económica Murcia-Orihuela en los finales del siglo XIV. "Anales de la Universidad de Alicanle. Historia Medieval 6 (en lo sucesivo AUA) (1987), pp. 311-324. Octavio GIL FARRt's, Historia de la moneda espariola, 1958, p. 142.

${ }^{58}$ A.c. 1380-VI-5, San Esteban de Gormaz., 1379-VII-2, Cartulario cra 1405-1418, f. 162 v. y 1380-VII-1, cartulario 1384-1391, f. 133 v.. A.c. 1386-VIII-23, XII-15, XII-19; Segovia, 1386-XI-24, cartulario 1384-1391, f. 140 r. y A.c. 1386-XII-22. Felipe MATEU Y LLOPIS, "De musatione inonetae" en el Reino de Valencia, Milano, 1962, p. 202. M" E. RUEDA SABATER, El florín, un "dolar" bajomedieval, "Estudios dedicados al Profesor Angel Ferrari", V,II. M* de los Llunos MART1NEZ CARRILLO, Revolución, p. 265.
} 
eran artesanos, algunos intermediarios entre el gran mercader y el consumidor, tragineros, tenderos, corredores, algunos grandes propietarios de ganados lanares y cuatro italianos tan solo, micer Francisco, micer Sorico Damar, micer Juan y micer Guido de la Vetula, que junto con los comerciantes de lana pago una de las multas mas altas; en total 265 afectados de una población de 8.000 habitantes $^{59}$. Los niveles adquisitivos regionales estaban muy por debajo de los precios, llegándose a extremos de auténtica subalimentación que estuvieron en la base de la guerra de bandos entre Manueles y Fajardos desrrollada entre 1391 y 1399. En general el monopolio de la oligarquia regional en la producción, importación y distribución del grano fue total y consecuencia de ello el hambre, la peste y el robo social que caracterizaron aquella década .

Las circustancias se prolongaron hasta la renovación de aranceles de Enrique III en 1403 que condujo a un cierre general de aduanas, que Torres Fontes interpretó como un intento estabilizador de la política de Castilla frente a sus vecinos; en 1409 se reabrieron los puertos tradicionales, que junto a la ciudad de Murcia eran Almansa, Yecla, Cartagena mas Alborea, con un desarrollo cuantitativo del valor de los arrendamientos del reino que pasó de 200.000 mrs. anuales en 1398 a 970.204 en 1421 , siendo muy significativo que el florentino Simon Destajo fuese el arrendador de las aduanas murcianas durante los primeros años del siglo $\mathrm{XV}$ y con él empezara a acentuarse el volúmen de las exportaciones de lana y grana procedentes de Murcia, en coincidencia con una serie de malas cosechas de cereal, generales también en Andalucía, as como de las importaciones de productos elaborados como los textiles, no necesariamente de lujo, que procedentes de largas distancias estaban sometidos a un tráfico que Iradiel califica de "para-colonial" 60.

\footnotetext{
${ }^{59} \mathrm{M}$ ' de los Llanos MARTINEZ CARRILLO, Las aduanas murcianas en el reinado de Enrique 11, "Homenaje al Profesor Juan Torres Fontes", Murcia, 1987, pp. 987-1004. Denis MENJOT, Le contrebande dans la marche frontiere murcienne au bas Moyen Age, "Homenaje al Profesor Juan Torres Fontes", pp. 1073-1083.

${ }^{\circ}$ Juan TORRES FONTES, La regencia de don Femando de Antequera. Poltrica exterior, "Anales de la Universidad de Murcia" (en lo sucesivo "AUM"), 1-2 (1959-1960), p. 41. Miguel Angel LADERO QUESADA, La Hacienda Real de Castilla en el siglo XV, La Laguna, 1973 , p. 103 y Instituciones fiscales y realidad socialo en el siglo XV castellano, "El siglo XV en Castilla. Fuentes de renta y política fiscal", Barcelona, 1982, pp. 66-67. Miguel Angel LADERO QUESADA y Manuel GONZALEZ JIMÉNEZ, Diezino eclesiástico y producción de cereales en el reino de Sevilla (1408-1513), Sevilla, 1979, pp. 38-39. $M^{*}$ de los Llanos MARTINEZ CARRILLo, Revolución, pp. 275-287. Paulino IRADIEL MURUGARREN, Estructuras
} 
Durante el siglo XV la falsificación de la moneda fue un factor estructural del comercio regional, que Juan II sanciono durante en 1434 con carácter general, siendo muy destacadas la serie de medidas adoptadas contra la ciudad de Murcia en la que el fenómeno resultaba endémico; escasez de moneda de vellón y duda sobre su legitimidad llevaron al concejo a pedir al rey la reapertura de una ceca que ya habra existido anteriormente en Murcia en tiempos de Alfonso XI y Enrique II y no se conseguirfa hasta $1466^{61}$.

Durante los reinados de Juan II y Enrique IV la moneda fuerte continuó siendo la aragonesa, lógica consecuencia de la utilización de la banca valenciana a la hora de buscar préstamos que en Murcia no se encontraban, utilizándose igualmente como moneda de cuenta; en 1449 se le pagaban a Diego de Mendoza " 13 florines catalanes de reales de Valencia, equivalentes a 1.035 mrs., por el precio de jaca de silla; en 1465 a la moneda aragonesa se le calificaba de "muy valariosa", siendo la causante de los altos precios agrícolas y ganaderos que se estaban dando, importándose el cereal de localidades como Albacete, Chinchilla, Peñas de San Pedro, Belmonte, Corral de Almaguer, Daimiel y la proxima Orihuel $a^{62}$ y hasta los genoveses exigran moneda aragonesa ${ }^{63}$, produciéndose en paralelo las crísis monetarias que mas afectaban al vellón con la especulación en el comercio de cereales,siendo entonces cuando mas se acudía al mercado sevillano para traer el trigo por mar hasta Cartagena.

Por la misma época, el contrahando monetario se seguía produciendo hacia Aragón, ası como la escasez de numerario en Murcia, sobre todo de blancas, por lo que en 1460 se fijaban los precios oficiales en la ciudad a los que tenía que atenerse el cambiador concejil "para desfacer, por la gran ganaçia que a los tales sacadores dello se sygue...", tijándosele las ganancias permitidas, que no variaron después de la reforma de Enrique

agrarias y modelos de organización industrial precapitalista en Castilla. "Sludist Histuricin. Hisiuria ivicuicuai", Salamanca, 1983, pp. 99-104.

${ }^{61}$ Segovia, 1434-IX-12, inserta en 1435-III-30, "CODOM", XVI, pp. to6 y tor. A. 1434-VIII-3I; 1435-VI-4, VI-14; 1440-I-22 y I-30. Juan TORKES FONTTIS, Le cecit musricists.s en el reinado de Alfonso XI, "Mélanges offerts à Jean Gautier-Dalché". Niv.A. 198.3. PW. 3(X) 307.

${ }^{62}$ A.c. 1444-II-27; 145I-VIII-3I; 1458-III-4; 1460-XI-29; 146I-V-2; 1464-IV-24; 147.3. VII-3I y 1482-VI-8.

${ }^{63}$ A.c. 1449-XI-4; 1465-I-8; 1466-VII-19 y 1472-VIII-22. Octavio GII. FARKl's, Hislıric de la moneda, p.220 
IV, a pesar del cambio a la haja de las monedas ${ }^{64}$; las eequivalencias de 1460 fueron estas:

-Enrique o noble de Inglaterra,de oro, 4 blancas.

-Dobla y corona castellanas, ducado veneciano y salute francés, de oro inferior, 3 blancas viejas.

- Florín de oro, timbre aragonés de oro que fue siendo sustituido por el tlorín a tines del XIV y dohla morisca, 2 blancas.

-Real castellano, de plata, una blanca vieja y real valenciano una blanca nueva.

Previamente a la reforma de Enrique IV, se pueden marcar tres niveles monetarios muy gráficos, que se corresponden con otros tantos niveles del comercio y el crédito marcados por Spyftords.

- Una moneda de oro en cada reino, por encima de las cuales estaban las "internacionales" de Venecia y Florencia; en el siglo XIV eran utilizadas para pagos principescos y en el XV se abrieron al gran comercio internacional, en Castilla la dobla hasta la primera acuñación de ducado en 1497.

- Moneda de plata usada para los principales pagos diarios, salarios, rentas e impuestos; en Castilla el real.

-Moneda de vellón que era la mas abundante en todas las ciudades y se utilizaba en las transacciones mas modestas y pequeñas compras alimenticias domésticas.

Dentro de la tendencia depresiva que caracterizó la baja edad media, Spuftord ha llamado "el triunfo del oro" a la época de la explotación de Kemnica en Hungría, que empezo a invadir Europa desde los años veinte del siglo XIV desplazando al oro musulmán que procedía de Al-Andalus; era mas ventajoso transportar reducidas cantidades de oro a grandes cantidades de plata y el negocio lo monopolizaron los venecianos. Por ello Ladero atirmaba que con anterioridad a las reformas de Enrique IV, los que disponían de oro poclían comprar mas productos cada vez, dado el descenso de los precios, siendo este el motor de los viajes a grandes distancias; sin embargo, a diferencia de sus antecesores Enrique IV en 1462 pretendió devaluar el oro y la plata y no el vellón, en perjuicio de los grandes banqueros y mercaderes; atirma, que en general provocó un gran colapso en

\footnotetext{
AtA.c. 1459-XII-18; 1460-I-5, VII-15 y 1461-V-30.

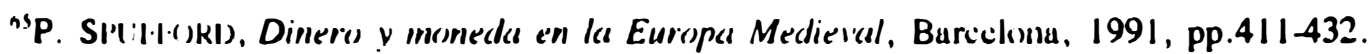


Andalucía y en centros mercantiles tan importantes como Toledo y Burgos, por lo que los nobles lo presionaron para rectificar y el resultado de todo ello fue la guerra civil que se inició inmediatamente sobre un inmenso caos económico ${ }^{66}$.

La sociedad murciana pasó de una depreciación fáctica de la moneda anterior a 1462, que el concejo se esforzaba en respaldar en su valor oficial a un intento de control sobre las de mas valor y mas usuales; sí antes de 1462 los reales de plata castellanos valían 20 mrs. y los aragoneses 15 , los carniceros no los aceptaban sí no era por una blanca menos de su valor oficial y en 1464 el concejo se esforzaba aún en hacer cumplir la normativa enriqueña, fijando el descuento que había que hacer en ambos, si despues de pesados estaban "menguados"67.

La realidad social era la falta de "moneda menuda", expuesta al rey como una necesidad de acuñar "moneda de oro e plata e villon altas de ley, e otras mas baxas para conprar e vender los mantenimientos e otras cosas de baxos presçios e para las limosnas", porque la población carecía de lo mas necesario y la reforma de 1462 estuvo precedida de una orden real de que las ciudades controlasen los precios de todo tipo de mercancias, desde viandas a ganados, paños, sedas, metalurgia, especiería... ${ }^{68}$. La tendencia regional fue la búsqueda de moneda de vellón procedente de Aragón a cambio de reales a un precio no oficial y la fragmentación de los propios reales para utilizarlos como vellón, con consecuencias negativas en el comercio a la altura de 1466, por ejemplo el vino, traido tanto de Aragón como de la

\footnotetext{
${ }^{66}$ Madrid, 1462-VI-9, "CODOM", XVIII, pp. 416419. P. SIIII/(1)kI) Diner(1, pp. 345350. A. MAC KAY, Las alteraciones inonelarias en la Ceastilla del sigelos XV: la inoneda de

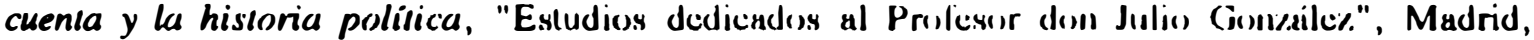

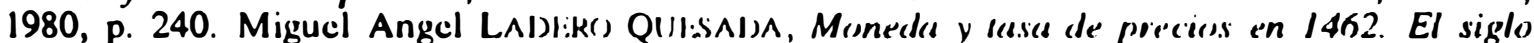

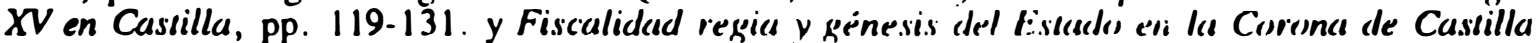
(1252-1504), "ETF" (Madrid, 1991), pp. 116-117.

${ }^{67}$ A.c. 1460-XII-16; 1464-VI-30 y X-13

${ }^{68}$ Aranda de Ducro, 1461-IV-24 y Madrid, 1462-VI 9, "CODOM", XVIII, pp. $349-365$ y 416-419. A.c. 1461-IX-22. La fijacion de precios y salarios locales en Murcia se hizo en 1462, Juan Tokris Fonlis, Estudios sobre la crinica de Enrique IV del Dr. Galindez de Carvajal, 1946, documento XXIV. M* Bclén PI()III:RAS GAR(IA, Fisculidad, real y, concejil en el reinado de Enrique IV, Cádiz. 1988, p. 173. Scrics de precios textiles en amplitud en Maria , La inclustria del vestido, pp. 39, 44, 61, 73, 84-85, 87, 89 y 93-94. Sohre los precios del carmero $M^{*}$ Angeles MARIN GARCiA, Las camicerias y el abastecioniento de carne en Murcia (1450-1500), p. 95. Precios varios entre 1450 y 1470 en José Dainián GONZAIJ:L ARCE, Inflacción y crisis. Tasas concejiles y lasas reales (Murcia /46()-1470), "MMM", XVI, pp. 96-99. Series de precios varios para el siglo XV en M" del Cannen VI:AS ARTlisli:R()S, La Hacienda concejil inurciana en el siglo XV (1423-1482), Murcia, microfonnu, 1988.
} 
Mancha durante toda la baja edad media; en Murcia se constata la bajada del precio de los cereales hasta llegar a ser un peligro de abandono de tierras cultivadas con renuncia a la vecindad, por lo que el concejo ordenó el consumo exclusivo del trigo cultivado en el regadío volviendo con ello a la mas pura autarquía, en contraste con los altísimos precios de los demás productos que quedaban fuera del alcance de la población llana en el mercado $^{69}$.

Escaseaba el pan y los pobres y forasteros no lo encontraban por su valor, las rentas particulares o concejiles descendieron en sus valores los años 1468-1475, que Torres Fontes califica de gobierno independiente el adelatado Pedro Fajardo ${ }^{70}$, lo cual repercute para el historiador en el empobrecimiento de los documentos emanados de la corte, advirtiéndose en las fuentes locales la proliferación de ordenanzas poniendo valor a las monedas que se emitran en la ceca murciana, as en 1471, que todos los enriques valiesen $17 \mathrm{mrs}$. por quilate; y fijando los precios de algunos productos como la carne, siempre en reales catalanes ${ }^{71}$, lo que prueba la implantación en la región de los mercaderes conectados con Valencia y la banca valenciana, así como los estímulos propiciados por el adelantado y la oligarquía que lo apoyaba para reactivar sus actividades.

En 1476 la batalla de Toro que aseguraba a los reyes en el trono de. Castilla, anunciaba el control de la nobleza, la consecuente actividad de Fajardo al servicio de la monarquía y la confirmación por parte de la reina del tradicional mercado franco, con dos años de franquicia en el pago de las alcabalas a todos los vecinos que quisiesen intercambiar las tierras que tenían en el término; tend́a a normalizar la situación de la década anterior social y económica, cuya mejor demostración fue la renuncia a tener ceca propia que el concejo hizo ante el adelantado "porque no es servicio de los reyes" ?? Las incertidumbres no desaparecieron por ensalmo; en 1480 al fijarse nuevos precios del oro y la plata, las blancas fundamentalmente, los tenderus murcianos y el concejo, pocas veces de acuerdo, se negaban a abrir los establecimientos "por la duda de la moneda" y lo mismo ocurría

\footnotetext{
${ }^{69}$ A.c. 1464-X-20, XI-13 y 1466-V-6. Maria MARTINEZ MARTINEZ, El comercio del vino aragonés en el mercado murciano, "XV Congreso de Historia de la Corona de Aragón" (en prensa).

${ }^{70 J u a n}$ TORRES FONTES, Don Pedro Fajardo, p. 109

"A.c. 1471-I-16, IV-9, V-9, V-14 y V-16.

${ }^{72}$ A.c. 1476-XII-31.
} 
en Valencia en las mismas fechas, "se feu la mutado dela moneda e ferense les reals nous e hagué moltes passions y congoxes"73. Otra vez los nuevos valores afectaban a rentas, salarios, impuestos y a los intercambios regionales y locales. Era el tipo de comercio que se daba predominantemente en el eje Murcia-Orihuela y su hinterland, sobre el que los mercaderes extranjeros hacian incisiones extractivas de materias primas de importancia creciente, que sin duda superaban en mucho al valor de las importaciones que solo afectaban a una minoría de privilegiados sociales y materiales. El cambio de rumbo en la región empezó a notarse desde 1483, con nuevas fluctuaciones climáticas, una serie de buenas cosechas bastante generales en el sur de Castilla y la posibilidad de exportar que ofrecian las guerras del Rosellón e Italia; el concejo murciano permitia la saca por el Mar Menor, prueba de que no habia escasez, y en Andalucia las cosechas estaban en la "normalidad" de la época, llegándose en los años noventa a ser posible la exportación a Barcelona ${ }^{74}$.

\section{CONCLUSIÓN}

Resumiendo, de los proyectos mediterráneos alfonsinos se pas 6 a la casi desconexión mercantil de la primera mitad del siglo XIV, salvo para el mercado de esclavos y el trigo, que eran lo únicos productos que interesaban a los mercaderes en el reino para operaciones concretas. A partir de la entronización de los Trastámara, la nueva y reducida oligarquía que se impuso en el territorio murciano, empezo a desarrollar las exportaciones en cantidades crecientes, y a abrir un reducidísimo mercado selectivo de importación, tanto por tierra como por mar, cuando las actividades del puerto de Cartagena siempre estuvieron subordinadas a los intereses de las grandes casas de banqueros y mercaderes, de acuerdo con la fluctuaciones impuestas por una sociedad de niveles sociales muy contrastados.

\footnotetext{
${ }^{73}$ Pregonada en Murcia en 1479, A.c. 1480-II-24, III-7 y VI-6. Felipe MATEU Y LLOPIS, De mulatione, p. 212.

${ }^{74}$ A.c. 1483-IX-20; 1495-VIII-18, XI-7 y 1500-X-3. Miguel Angel LADERO QUESADA y Manuel GONZALEZ JiMÉnEZ, Diezıno eclesiástico, p. 86. Relación de cosechas en Cayetano TORNEL COBACHO, El problema del trigo en Murcia en la época de los Reyes Católicos, "MMM", VI (1980), pp. 64-65. El problema ha sido estudiado fuera de la ciudad de Murcia en relación con el mercado regional, María MARTINEZ MARTINEZ, Producción y comercio de cereales en Lorca duranie la Baja Edad Media, "AEM", 19 (1989), pp. 640-641.
} 


\begin{abstract}
RÉSUMÉ
Le royaume de Murcie, après son incorporation définitive à la. Couronne de Castille en 1266 et la perte d'une partie de son territoire côtier en 1304-1305, participa avec difficulté aux activités méditerranéennes que le roi Alphonse $X$ avait cherché à travers les ports de Carthagene et d'Alicante. Pendant le XIV ${ }^{e}$ siecle il ne fut inclus que circonstanciellement dans les routes des grandes maisons de marchands italiens, et seule la conjonction de la faiblesse progressive de Grenade et les interêts d'exportation de laines et grains des grands propiétaires permit à partir la fin du XIV ${ }^{e}$ siècle et avec des difficultés, l'implantation de quelques maisons avec un certain degré de stabilité, en une constante relation avec le marché sévillan et la banque et l'artisanat de Valence. Cinq familles génoises en un peu plus d'un siecle canaliserent l'exportation des matieres premieres et l'importation des produits élaborés et de luxe en régime de monopole, qui, à côté des politiques monetaires, furent les facteurs fondamentaux de la stabilité, ou de l'instabilite. du marché.
\end{abstract}

\title{
SUMMARY
}

The kingdom of Murcia, after its final incorporation to the Castilian crown in 1266 as well as the loss of part of its coastal territory in 1304-1305, incorporated with some difficulties to the Mediterranean activites that Alfonso $X$ had searched through the ports of Cartagena and Alicante. During the XIV Century it was only circumstancially included into the routes of the big houses of Italian merchants, and just with the conjunction of the progressive weakness of Granada and the exporting interests of wool and seed of the hig proprietors, allowed, since the end of XIV Century and with difficulties, the implantation of some houses with a certain stability, in constant relation with the sevillian market and valencian banking and craftsmanship. In a little more time than a century, five Genoese families direc- 
ted the exportation of raw materials and the importation of luxury elaborated products as a monopoly system, that, along with the monetary policies, were the key factors of both stability and instability of the market. 